\title{
Comprehensive Quantification of the Spastic Catch in Children with Cerebral Palsy
}

Bar-On Lynn $\mathrm{PT}^{\mathrm{a}, \mathrm{c}}$, Aertbeliën Erwin Ing $\mathrm{PhD}^{\mathrm{b}}$, Molenaers Guy MD PhD ${ }^{\mathrm{a}, \mathrm{c}}$, Bruyninckx Herman Ing $\mathrm{PhD}^{\mathrm{b}}$, Monari Davide $\mathrm{Ing}^{\mathrm{b}}$, Jaspers Ellen $\mathrm{PhD}^{\mathrm{c}}$, Cazareck Anne $\mathrm{PT}^{\mathrm{c}}$, Desloovere Kaat $\mathrm{PhD}^{\mathrm{a}, \mathrm{c}}$

a. University Hospital, Pellenberg, Clinical Motion Analysis Laboratory, Weligerveld 1, 3212 Pellenberg, Belgium.

b. K.U. Leuven, Department of Mechanical Engineering, Celestijnenlaan 300b - box 2420, 3001 Heverlee, Belgium.

c. K.U. Leuven, Department of Rehabilitation Sciences, Tervuursevest 101, 3001 Heverlee, Belgium.

\begin{abstract}
In clinical settings, the spastic catch is judged subjectively. This study assessed the psychometric properties of objective parameters that define and quantify the severity of the spastic catch in children with cerebral palsy (CP). A convenience sample of children with spastic $\mathrm{CP}(\mathrm{N}=46$; age range, 4-16yrs) underwent objective spasticity assessments. High velocity, passive stretches were applied to the gastrocnemius (GAS) and medial hamstrings (MEH). Muscle activity was measured with surface electromyography (sEMG), joint angle characteristics using inertial sensors and reactive torque using a force sensor. To test reliability, a group of 12 children were retested after an average of $13 \pm 9$ days. The angle of spastic catch (AOC) was estimated by three biomechanical definitions: joint angle at (1) maximum angular deceleration; (2) maximum change in torque; and (3) minimum power. Each definition was checked for
\end{abstract}


reliability and validity. Construct and clinical validity were evaluated by correlating each AOC definition to the averaged root mean square envelope of EMG (RMS-EMG) and the Modified Tardieu Scale (MTS). Severity categories were created based on selected parameters to establish face validity. All definitions showed moderate to high reliability. Significant correlations were found between AOC3 and the MTS of both muscles and the RMS-EMG of the MEH, though coefficients were only weak. AOC3 further distinguished between mild, moderate and severe catches. Objective parameters can define and quantify the severity of the spastic catch in children with CP. However, a comprehensive understanding requires the integration of both biomechanical and RMS-EMG data.

Key words: Cerebral Palsy; Muscle Spasticity; Biomechanics; Electromyography

\section{Introduction}

Spasticity is the most common motor disorder in children with cerebral palsy (CP) (Cans et al., 2000). It alters muscle structure, may cause pain, and can hinder everyday activities such as gait. Lance (1980) defined spasticity as a motor disorder characterized by 'a velocity-dependent increase in the tonic stretch reflex'.

Clinicians assess spasticity by evaluating the level of resistance to passive muscle stretches (Schotles, Becher, Beelen, \& Lankhorst, 2006). Tardieu, Shentoub, and Delaru (1954) were the first to describe the concept of "spastic catch", i.e. a sudden reactive resistance elicited by increased muscle tone as a reaction to a fast passive stretch. Nowadays, the catch is commonly assessed by means of the Modified Tardieu Scale (MTS) (Boyd \& Graham, 1999). The velocitydependent stretch reflex is defined by the angle of the spastic catch (AOC), which is the difference between the end range of motion (ROM) while slowly moving the limb and the spastic 
catch angle while moving fast. A large AOC indicates increased spasticity (Boyd \& Graham, 1999). As the MTS integrates a velocity-dependent aspect, it closely relates to Lance's spasticity definition (1980) and is considered a valid tool to assess spasticity (Gracies et al., 2010; Mehhholz et al., 2005; Patrick \& Ada, 2006; Platz, Eickhof, Nuyens, \& Vuadens, 2005).

However, inconclusive intra- and inter-rater reliability of the MTS have been reported in children with CP (Haugh, Pandyan, \& Johnson, 2006). Similar to other clinical scales, the MTS relies on the subjective feeling of the therapist who stops the movement when a catch is felt (Platz et al., 2005). Van den Noort, Scholtes, and Harlaar (2009) additionally reported that goniometry, as used in the MTS, is an imprecise method to measure the AOC due to joint repositioning errors. Furthermore, the resistance felt at the spastic catch may comprise both the neural component of spasticity as defined by Lance (1980), and biomechanical components such as soft tissue compliance and joint integrity (Pandyan, Van Wijk, Stark, Vaudens, Johnson, \& Barnes, 2006).

Therefore, it is now widely acknowledged that more robust, quantitative measures to assess spasticity are required. Van den Noort et al. (2009) recommended the use of inertial sensors to measure joint angle characteristics more precisely. Others have shown that reactive torque can be objectively quantified using either hand-held dynamometers while manually stretching a spastic muscle (Akman et al., 1999; Lee, Chen, Ju, Lin, \& Poon, 2004; Pandyan, Price, Rodgers, Barnes, \& Johonson, 2001; Pandyan et al., 2006; Peng, Shah, Selles, GaeblerSpira, \& Zhang, 2004; Wu et al., 2010;) or using a motor-driven system to stretch the muscle (Damiano, Laws, Carmines, \& Abel, 2006; Engsberg, Ross, Orlee, \& Park, 2000; Wood et al., 2005).

Although these biomechanical methods provide more quantitative results compared to the clinical methods (Wood et al., 2005), a valid tool for spasticity assessment should also include 
simultaneous measurement of muscle activity (Lance, 1980; Malhotra, Pandyan, Rosewilliam, Roffe, \& Hermens, 2011; Voerman, Gregoric, \& Hermens, 2005; Wood et al., 2005). In children with $\mathrm{CP}$, muscle activity is most commonly measured using surface electromyography (sEMG) (Voerman et al., 2005). A comprehensive spastic catch measurement based on biomechanical signals and sEMG would capture: (1) an augmentation in muscle activity that corresponds to an increase in sEMG amplitude, (2) a consequent reactive torque to passive stretch, and (3) a change in joint angle characteristics (Calota, Feldman, \& Levin, 2008; van den Noort, Scholtes, Becher, \& Harlaar, 2010). This will allow for a more objective definition of the spastic catch.

Thus far, only two papers have reported sEMG measurements combined with biomechanical parameters to objectively define the AOC. Van den Noort, Schotles, Becher, and Harlaar (2010) evaluated the AOC in the gastrocnemius and medial hamstrings in children with spastic CP using sEMG and inertial sensors. They defined the catch as the angle corresponding to the time of maximum joint deceleration when the joint is passively moved at high velocity. Wu et al. (2010) objectively measured the AOC in the elbow flexors of children with CP using sEMG and a manually controlled device equipped with torque and angle sensors. They defined the catch as the angle corresponding to the time of maximum change in torque.

Although these studies provided a more objective spasticity assessment compared to the MTS, the psychometric properties of these methods warrant further investigation. Furthermore, these studies used the position of the catch as the measure to quantify the severity of spasticity, whereas $\mathrm{Wu}$ et al. (2010) have shown that this position is dependent on the stretch velocity and may therefore not solely reflect severity. Finally, the definitions for the AOC were predominantly based on isolated signals (velocity - van den Noort et al., 2010; or torque - Wu et al., 2010) and a more valid measure of a spastic catch includes the integration of signals (Wood et al., 2005). 
In line with a multi-dimensional assessment method, it is believed that the integration of signals may provide an improved quantification of the AOC. The aim of this study was to investigate the reliability and validity of an objective spastic catch assessment. Biomechanical and sEMG parameters obtained from isolated and integrated signals, that could define the AOC and quantify the catch severity, were investigated.

\section{Methods}

\subsection{Participants}

Children with CP were recruited from the database of the Clinical Motion Analysis Laboratory (University hospital*blinded for review*). Participants were eligible for inclusion if they were diagnosed with spastic $\mathrm{CP}$ and aged between 4 and 18 years. They were excluded in case of: (1) presence of ataxia or dystonia; (2) severe muscle weakness (score $<2+$ on the Manual Muscle Test - Sapega, 1990) and/or poor selectivity (Gage, 2004); (3) ROM less than 25\% of the normal values for all lower limb joints (based on clinical examination); (4) cognitive problems that could hinder communication and cooperation during the assessment; (5) treatment with botulinum toxin type-A six months prior to assessment or previous lower limb orthopedic surgery, intrathecal baclofen or selective dorsal rhizotomy. Written informed consent for participation was sought from all parents. The experimental protocol was approved by the local Ethical Committee.

\subsection{Measurement protocol}

Prior to the objective spasticity assessment, all participants underwent a full lower limb clinical examination, including the Modified Ashworth Scale (MAS) (Bohannon \& Smith, 1987) 
of the gastrocnemius (GAS) and medial hamstrings (MEH). Muscles with a MAS-score of 1+ or higher were additionally assessed with the MTS (Boyd \& Graham, 1999). In children with unilateral $\mathrm{CP}$, the objective spasticity assessments were carried out on the affected side only. In children with bilateral CP, the most affected side was tested (highest average MAS for GAS and MEH). In participants with symmetrical MAS-scores, the side with a more severe MTS was selected.

All measurements were performed by one trained assessor. The protocol for the objective spasticity assessment included: preparation, maximum voluntary isometric contractions (MVIC), joint calibrations and passive stretches (setup shown in Fig. 1). Muscle activity was measured using sEMG at a sample rate of $2000 \mathrm{~Hz}$ (Zerowire: Cometa, Milan, IT). Circular Ag/AgCl electrodes (diameter of $2 \mathrm{~cm}$ ) were placed on the muscle bellies of MEH and GAS, as well as on the antagonists of these muscles (rectus femoris and tibialis anterior, respectively) with an interelectrode distance of 2cm (Hermens, Freriks, Diseelhorst-Klug, \& Rau, 2000).

To define joint angles, angular velocity and acceleration, two inertial measurement units (IMUs; Analog Devices, ADIS16354) were used to track the movement of the distal limb segment with respect to the proximal segment. The IMUs were placed arbitrarily on the lower limb so as not to interfere with the position of other sensors. Joint calibration trials (predefined reference positions and motions) were performed prior to the passive stretches to compute anatomical joint angles from the IMUs. Reactive torque was measured using a six degrees of freedom force-sensor load-cell (ATI mini45: Industrial Automation). The force-sensor was attached to the segment using a light-weight foot or shank orthosis for the GAS and the MEH respectively (Figure 1 and Appendix A). Motion and torque were sampled at 200Hz.

Segment lengths (lower leg and foot length) and the perpendicular distances (moment arms) between the joint axes and the z-axis of the load-cell were determined with measuring-tape. 
During the passive stretch trials, muscles were tested by manually moving the joints from a predefined starting position through their full ROM, first slowly during 5 seconds, then at medium velocity and finally as fast as possible. Each stretch trial was repeated four times with an interval of seven seconds rest between repetitions. The end position of each stretch was maintained for a period of three seconds.

\subsection{Research design}

Between-session reliability and validity of the parameters derived from the objective spasticity assessment were tested. Twelve children, who were willing to be tested twice, were reevaluated within four weeks and results from the first test session were used to assess validity. Validity was examined on three aspects: construct, clinical and face validity (Mokkink et al., 2010). An overview of how psychometric properties were defined and assessed can be found in Table 1.

\subsection{Data analysis}

Data of the participants was processed using customized software (MATLAB 7.6.0 R2008a: MathWorks). The raw sEMG signal was filtered with a $6^{\text {th }}$ order zero-phase Butterworth bandpass filter from 20 to $500 \mathrm{~Hz}$. Next, the root mean square envelope of the sEMG (RMSEMG) signal was extracted by applying a low-pass $30 \mathrm{~Hz} 6^{\text {th }}$ order zero-phase Butterworth filter on the squared raw sEMG signal. To estimate joint angle characteristics, a Kalman smoother (Rauch, Tung, \& Striebel, 1965) was applied on the data obtained from the IMUs. Torque signals were filtered using a low-pass filter with a cut-off frequency of $50 \mathrm{~Hz}$ (Wu et al., 2010). The net internal joint torque was calculated from the segment lengths and moment arms, the external 
forces, the exerted moments, and the forces caused by gravity and inertia (Jensen, 1986). See Appendix A for a detailed overview of the different torque components.

Only data collected during passive stretches at the highest velocity were examined as these were expected to best reflect the spastic catch. Per muscle, the first well-performed stretch at high velocity with an sEMG onset was used for analysis. Well-performed stretches constituted stretches that had a comparable velocity between repetitions. sEMG onset was defined as the time of first muscle activity according to the Staude method (Staude \& Wolf, 1999). Furthermore, trials were deleted in case of simultaneous antagonist activity, active assistance of the subject or in case of poor quality sEMG signal (low signal-to-noise ratio or clear artifacts).

\subsection{Outcome Parameters}

Two quantitative measures for AOC (AOC-definitions) were developed based on the isolated signals of either angular velocity $(\omega)$ or torque (T): AOC1 was defined as the position of maximum deceleration (van den Noort et al., 2010); AOC2 was defined as the position of maximum change in torque (dT/dt) (Wu et al., 2010). A third AOC-definition (AOC3) combined signals from velocity and torque, i.e. the angular position corresponding to the first local minimum of power after a local maximum of power. Power was defined as the product of angular velocity and torque $\left(\omega^{*} \mathrm{~T}\right)$. All three AOC-definitions were expressed in percentages that indicated the relative position of the AOC with respect to the ROM of the same trial.

Other severity related parameters included: maximum dT/dt $(\mathrm{Nm})$, maximum deceleration $\left(\% / \mathrm{sec}^{2}\right)$, minimum power $(\mathrm{W})$ and, work $(\mathrm{J})$. Work was defined as the area under the power-time curve over a given interval. The time interval started from the time corresponding to maximum velocity and ended at the time corresponding to an angular position ( $90 \%$ of the ROM). Average RMS-EMG was also explored as a severity-related parameter. Average RMS-EMG was 
calculated by dividing the area under the RMS-EMG-time curve by the duration of the sEMG onset (Staude \& Wolf, 1999) and expressed as a percentage of the peak RMS-EMG value of three MVIC repetitions.

\subsection{Statistical analysis}

Between-session reliability for GAS and MEH was tested with the Intraclass Correlation Coefficients ( $\left.\mathrm{ICC}_{1,1}\right)$ with 95\% confidence intervals (McGraw \& Wong, 1996) and the standard error of measurement (SEM). The SEM was calculated from the square root of the mean square error from one-way ANOVA (Weir, 2005). SEM values were further expressed as a percentage of the mean of the parameter. ICC-values $\geq 0.8$ indicated high; $\geq 0.6$ moderately high; and $\geq 0.4$ moderate reliability (Katz, Larsen, Philips, Fossel, \& Liang, 1992).

For the validity studies, Spearman rank correlation coefficients were calculated between the different outcome parameters for each muscle. To assess construct validity (Mokkink et al., 2010) correlations between RMS-EMG, maximum dT/dt, maximum deceleration, minimum power, work, and the AOC definitions were calculated. Clinical validity was tested by correlating the outcome parameters to the MTS (Boyd \& Graham, 1999). Correlation values of $>0.7$ indicated a high, $0.50-0.70$ a good, $0.30-0.50$ a weak and $<0.30$ a poor correlation (Hinkle, Wiesma, \& Jars, 1994).

Face validity was established using the following visual categorization. Based on results of reliability and construct validity of the three AOC definitions, the position-time graph, the power-time graph and the RMS-EMG-time graph were visualized to categorize the severity of the spastic catch. According to a predefined workflow, two assessors independently categorized each muscle as 1) no or mild; 2) moderate; or 3) severe spastic catch. Fig. 2 shows an example of the 
reasoned workflow to interpret the severity of the spastic catch in the GAS. In the absence of a catch or in a mild catch, AOC3 was detected within the last $20 \%$ of the ROM, the change in direction of the power was $\leq 10 \%$ of the maximum power, the RMS-EMG amplitude was negligible with respect to baseline activity and/or the RMS-EMG onset duration was $\leq 0.03$ seconds. In a severe catch, AOC3 occurred earlier in the ROM, minimum power was negative and the RMS-EMG amplitude was $\geq 5$ SD than baseline. Catches were considered moderate when they did not fall into any of the latter two categories. When the two assessors did not reach consensus, a third and independent assessor reclassified the trial. In those cases, the majority opinion decided the severity of the catch.

Percentage agreement was calculated between the assessors for each muscle. KruskalWallis tests (KW-test) were applied to verify whether a parameter could significantly distinguish between the three created severity groups, with post-hoc Mann-Whitney U tests (MW U-test). Statistical analyses were carried out in Statistica (version 10). Significance level was set at 0.05.

\section{Results}

Fifty-one participants were tested with the objective spasticity assessment. Five participants were excluded (mean age 7.72yrs; range 4.39yrs - 11.67yrs; four girls, one boy); two participants because there was no EMG onset in their GAS, and three participants due to poor quality EMG data. Subject characteristics of the remaining 46 participants are presented in Table 2. For ca. $20 \%$ of these participants, the first high velocity stretch did not fulfill the performance criteria and the next well-performed stretch was selected for further analysis. MTS-scores were noted in 44 participants for the GAS and in 35 participants for the MEH.

Twelve participants were re-tested after an average of $13 \pm 9$ days and included in the reliability study. No significant differences in age, weight, gender, average MAS- and MTS- 
scores, Gross Motor Function Classification System (GMFCS) (Palasino et al., 1997) or diagnosis was found between participants included in the reliability study and the total group (Table 2).

\subsection{Reliability}

Outcomes of the reliability study are presented in Table 3. The majority of the parameters showed moderately high to high between-session reliability. Maximum deceleration and RMSEMG for the GAS and minimum power for the MEH were moderately reliable. Among the three AOC-definitions, AOC3 showed highest ICCs for both muscles (range 0.62-0.86) and lowest $\%$ SEM values (range 5.40\%-15.77\%). The majority of the severity-related parameters showed SEM values ranging from $16.1 \%-25.8 \%$ of the mean with the exceptions of minimum power for both muscles (GAS 62.0\%; MEH 75.2\%) and RMS-EMG for the GAS (73.5\%) which showed high \%SEM values.

\subsection{Validity}

An overview of the correlations between the outcome parameters and between outcome parameters and the MTS can be found in Table 4. In both muscles, poor correlations were found between maximum deceleration and maximum dT/dt (GAS r=0.021; MEH r= -0.13). In the GAS, weak correlations were also found between work and RMS-EMG $(r=-0.30)$ and between maximum dT/dt and RMS-EMG (r=-0.37). In both muscles, all three AOC definitions were significantly correlated to each other, ranging from good to high for the GAS and from weak to high for the MEH. Work was correlated to all three AOC definitions in the GAS, and to AOC1 and AOC3 in the MEH. In the MEH, the correlation to RMS-EMG was highest for AOC3 (r=$0.65)$. 
Of the three AOC definitions, only AOC3 showed significant, but weak correlations with the MTS in both muscles (GAS r=0.31; MEH r=0.34). In the MEH significant, but weak correlations were also found between maximum dT/dt, work, RMS-EMG and the MTS.

Of the three AOC definitions, AOC3 showed the highest relative reliability, lowest measurement error and the highest clinical validity for both muscles. In the $\mathrm{MEH}$, it also established the highest correlation to RMS-EMG thus confirming its construct validity. Therefore, it was decided to use this AOC definition, and the power-time graph, to visually categorize the muscles into severity groups. Due to the overall poor correlations between outcome parameters and RMS-EMG, it was decided to further use the RMS-EMG for categorization.

Agreement between assessors on visual categorization was $91.80 \%$ for the GAS and $80.33 \%$ for the MEH. For the GAS, 16 muscles were categorized as having no or mild catch, 22 as having a moderate catch and eight as having a severe catch. For the MEH, 12 muscles were categorized with no or mild catch, 21 with a moderate catch and 12 with a severe catch. Fig. 3 shows the results of the comparison of selected parameters between severity categories. AOC3 and minimum power were significantly different between the visually defined severity categories for both the GAS $(p<0.001 ; p<0.001$, respectively) and the MEH $(p<0.001 ; p=0.002$, respectively). In the GAS, AOC3 was not able to distinguish between moderate and severe catches $(p=0.62)$. In the MEH however, AOC3 could distinguish between no/mild and moderate $(p=0.004)$ as well as between moderate and severe $(p=0.003)$.

In the MEH, minimum power could distinguish between moderate and severe catches $(p<0.001)$ but not between no/mild and moderate catches ( $p=0.84)$. RMS-EMG was also significantly different between the categories $(p=0.007)$ in the MEH, but could only distinguish between no/mild and severe catches $(p=0.006)$. 


\section{Discussion}

The aim of the current study was to explore the psychometric properties of isolated, as well as integrated signals to define and quantify the severity of the spastic catch in the MEH and GAS in a heterogeneous group of children with spastic CP. To date, this is the first methodological study to check the reliability and validity of such objective AOC definitions and severity indicators. The ability of the instrument to measure a variety of participants reliably, points towards its value for future in-depth explorations of the possible causes of the spastic catch and important influencing factors, i.e. age, diagnoses and functional level. Such insights will contribute to patient categorization and can improve treatment delineation.

All AOC definitions were found reliable. However, the lack of correlation between the individual signals, used to compose the AOC, highlighted the need to integrate parameters to achieve proper construct validity of the AOC as a severity indicator. The definition of AOC3, i.e. AOC based on minimum power, represented the best definition of the spastic catch, since both torque and velocity were considered. In the $\mathrm{MEH}, \mathrm{AOC} 3$ was also significantly correlated to RMS-EMG, which further confirms its improved construct validity with respect to the other two definitions (AOC1 and AOC2). For the GAS however, AOC3 alone was not able to distinguish between moderate and severe catches. By additionally examining the value of the minimum power, moderate and severe catches could be distinguished. In the $\mathrm{MEH}$, face validity of AOC3 was established as it could distinguish between all three severity groups. Nonetheless, the low correlations between minimum power and RMS-EMG emphasize the need to additionally examine sEMG parameters to further quantify the severity of the AOC and relate it to Lance's definition of spasticity (Lance, 1980). Previous studies have also indicated the need to assess 
muscle activity in order to quantify spasticity (Calota et al., 2008; Damiano et al., 2002; Malhotra et al., 2011; Pandyan et al., 2006).

The MTS is a commonly used clinical test to measure spasticity and, given the lack of a golden standard for quantifying the AOC, may be a useful and fast method to screen for spasticity or provide rough clinical validity of an instrumented alternative. However, several sources of error may be introduced while using the MTS. First, the velocity of stretch is not controlled. An instrumented evaluation however, can report on the velocity and performance of the stretch. Such information improves the standards of clinical practice and allows for more accurate interpretation of results.

Second, the passive stretch was performed over the full ROM and the end position was held for three seconds, contrary to the MTS. This technique, which was also applied by Wu et al. (2010), minimized the influence of performance-related errors by removing the subjective decision of the examiner to stop the movement. However, in muscles with very high resistance (e.g. MAS $\geq 3$ ), the end position of the stretch may have been underestimated which could have affected the AOC calculations. In such cases, additionally examining minimum power and the amount of RMS-EMG may prove more useful in determining the catch severity than the relative AOC position alone.

Interestingly, Wu et al. (2010) also found that the AOC is sensitive to the velocity of the passive stretch with later AOC positions being achieved with increasing stretch velocity. This finding is in contrast to the hypothesis that higher stretch velocities elicit a stronger hyperactive stretch reflex and thus an earlier appearance of the spastic catch. Therefore, assessing the spastic catch based on the catch position alone may not adequately reflect the construct to be assessed.

Similar to the findings of Alhousaini et al. (2010), a low correlation was found between the MTS-score and RMS-EMG, especially for the GAS. The MTS is thus less likely to be 
associated with electrophysiological parameters and therefore cannot be considered a valid measure of spasticity (Lance, 1980). These findings indicate the importance of measuring the velocity-dependent hyperactive stretch reflex, which is lacking in current clinical tests (Damiano et al., 2002). Therefore, it is recommended that, especially for children undergoing treatment, multidimensional signals should be integrated when measuring the spastic catch. An instrumented assessment provides more informative, continuous, and valid data which may prove more sensitive in distinguishing those patients that benefit best from anti-spasticity treatment.

Although the objective spastic catch assessment was found reliable and valid, some study limitations need to be considered. Firstly, in spite of the seven second-rest between repetitions to avoid post activation depression, in some cases, the AOC occurred later in the ROM with repeated stretches. This may be due to the mechanical effect of stretching a muscle (Voerman et al., 2005). To control this effect, the first, well-performed repetition per muscle was analyzed as it was considered to be the best representation for the severity of the spastic catch. In case the first stretch was not properly executed (+/- in $20 \%$ of the participants), the next well-performed stretch was chosen, which may have resulted in some muscles being categorized as having less severe catches.

Secondly, results showed low reliability and high measurement error for minimum power. This highlights that despite the standardization of the movement, outcome parameters still depend on how the stretch is performed. While motor-controlled isokinetic devices allow to displace a limb at a controlled torque and velocity, Rabita et al. (2005) have shown that such isokinetic devices cannot be used to simulate manual spasticity tests as the mobilization characteristics are very different. These authors found that the stretch reflexes elicited by a manually-applied transient acceleration could not be generated by the constant acceleration of an isokinetic device (Rabita et al., 2005). Manual stretching could however be improved by providing the examiner 
with feedback about the consistency of the force applied during the repetitions and by following a pre-defined tracking signal while stretching (Wood et al., 2005; Wu et al., 2010).

Spasticity was determined by means of the MAS, following routine clinical test procedures. However, the MAS has been criticized for not being a valid measure of spasticity (Fleuren, Voerman, \& Erren-Wolters, 2010). Indeed, three patients with MAS 0 in the MEH had an EMG onset during passive stretch and were visually classified as having a mild catch.

Conversely, two patients with spasticity in the GAS (MAS1+ and MAS2) were excluded because no EMG activity was evoked when stretching at high velocity. The value of the MTS as an indicator for the presence or absence of spasticity should be further explored.

The proposed method cannot be used to distinguish other spasticity phenomena, such as a clonus, from a catch. An algorithm that can automatically identify exceptions, prior to categorizing the catches, should be developed. Lindenberg et al. (2001) distinguished reflex mediated resistance (neural component) from elastic/viscous resistance (non-neural component) by measuring torque during stretches of finger and wrist flexors at low and high velocity. In the current study, it was found that the biomechanical parameters of the GAS were less related to RMS-EMG than in the MEH. Catches in the GAS may be more influenced by altered muscular and soft tissue structure than by the pure velocity-dependent stretch reflexes (Foran, Steinman, Barash, Chambers, \& Lieber, 2005).

More severely involved children with CP did not meet the inclusion criteria of the current study. As a result, only three participants with quadriplegia were included in the total study sample. Future work should focus on developing spastic catch assessment methods that are appropriate for children with higher GMFCS scores and/or with more severe muscle weakness.

Finally, the amount of sEMG is very susceptible to inter-subject variability. In the current study, the RMS-EMG was therefore normalized with respect to the muscles' MVIC. However, 
the MVIC may be difficult to collect in children with CP (Phadke, Ismail \& Boulias, 2012) and requires strict inclusion criteria. Alternative normalization methods, e.g. normalization to muscle activity during a known force, tended to produce more variable results while others were considered too invasive for use in children e.g. M-wave excitation. Alternatively, future studies could establish the sensitivity of additional parameters, such as velocity and position at EMG onset that prevent the need for normalization.

\section{Conclusions}

Objective definition and quantification of the severity of the spastic catch requires integrated signal parameters. This study proposes to take the AOC, defined by the time of the minimum power, the minimum power value itself, as well as RMS-EMG in order to define the spastic catch and measure its severity.

\section{Acknowledgments}

This work was made possible by a grant from the Doctoral Scholarships Committee for International Collaboration with non EER-countries (DBOF) of the Katholieke University of Leuven, Belgium. This work was further supported by a grant from for Applied Biomedical Research from the Flemish agency for Innovation by Science and technology (IWT-TBM: grant number 060799); and by 'Move-to-improve', a special support funding in Flanders for children with motor problems (http://www.movetoimprove.org).

\section{Appendix A. Supplementary data}




\section{References}

Akman, M. N., Bengi, R., Karatas, M., Kilinc, S., Sozay, S., \& Ozker, R. (1999). Assessment of spasticity using isokinetic dynamometry in patients with spinal cord injury. Spinal Cord, 37, 638-643.

Alhusaini, A. A. A., Dean, C. M., Crosbie, J., Shepherd, R. B., Lewis, J. (2010). Evaluation of spasticity in children with cerebral palsy using Ashworth and Tardieu scales. Journal of Child Neurology, 25, 1242-1247.

Bohannon, R. W. \& Smith, M. B. (1987). Interrater reliability of a Modified Ashworth Scale of muscle spasticity. Physical Therapy, 67, 206-207.

Boyd, R. N. \& Graham, H. K. (1999). Objective measurement of clinical findings in the use of botulinum toxin type A for the management of children with cerebral palsy. European Journal of Neurology, 6, S23-S35.

Calota, A., Feldman, A. G., \& Levin, M. F. (2008). Spasticity measurement based on tonic stretch reflex threshold in stroke using a portable device. Clinical Neurophysiology, 119, 2329-2337.

Cans, C., Guillem, P., Baille, F., Arnaud, C., Chalmers, J., Cussen, G. et al. (2000). Surveillance of cerebral palsy in Europe: a collaboration of cerebral palsy surveys and registers. Developmental Medicine and Child Neurology, 42, 816-824. 
Damiano, D. L., Quinlivan, J. M., Owen, B. F., Payne, P., Nelson, K. C., \& Abel, M. F. (2002). What does the Ashworth scale really measure and are instrumented measures more valid and precise? Developmental Medicine and Child Neurology, 44, 112-118.

Damiano, D. L., Laws, E., Carmines, D. V., \& Abel, M. F. (2006). Relationship of spasticity to knee angular velocity and motion during gait in cerebral palsy. Gait \& Posture, 23, $1-8$.

Engsberg, J. R., Ross, S. A., Olree, K. S., \& Park, T. S. (2000). Ankle spasticity and strength in children with spastic diplegic cerebral palsy. Developmental Medicine and Child Neurology, 42, 42-47.

Fleuren, J. F. M., Voerman, G. E., Erren-Wolters, C. V., Snoek, G. J., Rietman, J. S., Hermens, H. J. et al. (2010). Stop using the Ashworth Scale for the assessment of spasticity. Journal of Neurology Neurosurgery and Psychiatry, 81, 46-52.

Foran, J. R. H., Steinman, S., Barash, I., Chambers, H. G., \& Lieber, R. L. (2005). Structural and mechanical alterations in spastic skeletal muscle. Developmental Medicine and Child Neurology, 47, 713-717.

Gage, J. R. (2004). The treatment of gait problems in cerebral palsy. London: Mac Kieth Press.

Gracies, J. M., Burke, K., Clegg, N. J., Browne, R., Rushing, C., Fehlings, D. et al. (2010). Reliability of the Tardieu Scale for Assessing Spasticity in Children With Cerebral Palsy. Archives of Physical Medicine and Rehabilitation, 91, 421-428. 
Haugh, A. B., Pandyan, A. D., \& Johnson, G. R. (2006). A systematic review of the Tardieu Scale for the measurement of spasticity. Disability and Rehabilitation, 28, 899-907.

Hermens, H. J., Freriks, B., Disselhorst-Klug, C., \& Rau, G. (2000). Development of recommendations for SEMG sensors and sensor placement procedures. Journal of Electromyography and Kinesiology, 10, 361-374.

Hinkle, D. E., Wiersma, W., \& Jars, S. G. (1994). Applied Statistics for the Behavioral Sciences. (3 ed.) Boston: Houghton Miffin Company.

Jensen, R. K. (1986). Body Segment Mass, Radius and Radius of Gyration Proportions of Children. Journal of Biomechanics, 19, 359-368.

Katz, J. N., Larson, M. G., Philips, C. B., Fossel, A. H., \& Liang, M. H. (1992). Comparing measurement sensitivity of short and longer health status instruments. Medical Care, 30, 917-925.

Lance, J. W. (1980). Pathophysiology of spasticity and clinical experience with baclofen. In Feldman, R. G; Young, R. R, \& Koella, W. P. (Eds.) Spasticity: disordered motor control. Chicago: Yearbook Medical.

Lee, H. M., Chen, J. J. J., Ju, M. S., Lin, C. C. K., \& Poon, P. P. W. (2004). Validation of portable muscle tone measurement device for quantifying velocity-dependent properties in elbow spasticity. Journal of Electromyography and Kinesiology, 14, 577-589.

Lindberg, P. G., Gaverth, J., Islam, M., Fagergren, A., Borg, J., \& Forssberg, H. (2011). Validation of a New Biomechanical Model to Measure Muscle Tone in Spastic Muscles. Neurorehabilitation and Neural Repair, 25, 617-625. 
Malhotra, S., Pandyan, A. D., Rosewilliam, S., Roffe, C., \& Hermens, H. (2011). Spasticity and contractures at the wrist after stroke: time course of development and their association with functional recovery of the upper limb. Clinical Rehabilitation, 25, 184-191.

Mcgraw, K. O. \& Wong, S. P. (1996). Forming inferences about some intraclass correlation coefficients. Psychological Methods, 1, 30-46.

Mehrholz, J., Wagner, K., Meissner, D., Grundmann, K., Zange, C., Koch, R. et al. (2005). Reliability of the Modified Tardieu Scale and the Modified Ashworth Scale in adult patients with severe brain injury: a comparison study. Clinical Rehabilitation, 19, 751-759.

Mokkink, L. B., Terwee, C. B., Patrick, D. L., Alonso, J., Stratford, P. W., Knol, D. L. et al. (2010). The COSMIN study reached international consensus on taxonomy, terminology, and definitions of measurement properties for health-related patient-reported outcomes. Journal of Clinical Epidemiology, 63, 737-745.

Palisano, R., Rosenbaum, P., Walter, S., Russell, D., Wood, E., \& Galuppi, B. (1997). Development and reliability of a system to classify gross motor function in children with cerebral palsy. Developmental Medicine and Child Neurology, 39, 214-223.

Pandyan, A. D., Price, C. I. M., Rodgers, H., Barnes, M. P., \& Johnson, G. R. (2001). Biomechanical examination of a commonly used measure of spasticity. Clinical Biomechanics, $16,859-865$.

Pandyan, A. D., Van Wijck, F. M., Stark, S., Vuadens, P., Johnson, G. R., \& Barnes, M. P. (2006). The construct validity of a spasticity measurement device for clinical practice: an alternative to the Ashworth scales. Disability and Rehabilitation, 28, 579-585. 
Patrick, E. \& Ada, L. (2006). The Tardieu Scale differentiates contracture from spasticity whereas the Ashworth Scale is confounded by it. Clinical Rehabilitation, 20, 173-182.

Peng, Q., Shah, P., Selles, R. W., Gaebler-Spira, D. J., \& Zhang, L. Q. (2004). Measurement of Ankle spasticity in Children with Cerebral Palsy using a manual Spasticity Evaluator. In IEEE Engineering in Medicine \& Biology Society (pp. 4896-4899).

Phadke, C. P., Ismail, F., \& Boulias, C. (2012). Assessing the neurophysiological effects of botulinum toxin treatment for adults with focal limb spasticity: a systematic review. Disability and Rehabilitation, 34, 91-100.

Platz, T., Eickhof, C., Nuyens, G., \& Vuadens, P. (2005). Clinical scales for the assessment of spasticity, associated phenomena, and function: a systematic review of the literature. Disability and Rehabilitation, 27, 7-18.

Rabita, G., Dupont, L., Thevenon, A., Lensel-Corbeil, G., Perot, C., \& Vanvelcenaher, J. (2005). Differences in kinematic parameters and plantarflexor reflex responses between manual (Ashworth) and isokinetic mobilisations in spasticity assessment. Clinical Neurophysiology, 116, 93-100.

Rauch, H. E., Tung, F., \& Striebel, C. T. (1965). Maximum Likelihood Estimates of Linear Dynamic Systems. American Institute of Aeronautics and Astronautics Journal; 3, 14451450.

Sapega, A. A. (1990). Muscle Performance Evaluation in Orthopedic Practice. Journal of Bone and Joint Surgery-American Volume, 72A, 1562-1574. 
Scholtes, V. A. B., Becher, J. G., Beelen, A., \& Lankhorst, G. J. (2006). Clinical assessment of spasticity in children with cerebral palsy: A critical review of available instruments. Developmental Medicine and Child Neurology, 48, 64-73.

Staude, G. \& Wolf, W. (1999). Objective motor response onset detection in surface myoelectric signals. Medical Engineering \& Physics, 21, 449-467.

Tardieu, G., Shentoub, S., \& Delaru, R. (1954). A la recherche d'une technique de measure de la spasticite. Revue neurologique (Paris), 91, 143-144.

van den Noort, J. C., Scholtes, V. A., \& Harlaar, J. (2009). Evaluation of clinical spasticity assessment in Cerebral palsy using inertial sensors. Gait \& Posture, 30, 138-143.

van den Noort, J. C., Scholtes, V. A., Becher, J. G., \& Harlaar, J. (2010). Evaluation of the Catch in Spasticity Assessment in Children With Cerebral Palsy. Archives of Physical Medicine and Rehabilitation, 91, 615-623.

Voerman, G. E., Gregoric, M., \& Hermens, H. J. (2005). Neurophysiological methods for the assessment of spasticity: The Hoffmann reflex, the tendon reflex, and the stretch reflex. Disability and Rehabilitation, 27, 33-68.

Weir, J. P. (2005). Quantifying test-retest reliability using the intraclass correlation coefficient and the SEM. Journal of Strength and Conditioning Research, 19, 231-240.

Wood, D. E., Burridge, J. H., Van Wijck, F. M., Mcfadden, C., Hitchcock, R. A., Pandyan, A. D. et al. (2005). Biomechanical approaches applied to the lower and upper limb for the measurement of spasticity: A systematic review of the literature. Disability and Rehabilitation, 27, 19-32. 
Wu, Y. N., Ren, Y. P., Goldsmith, A., Gaebler, D., Liu, S. Q., \& Zhang, L. Q. (2010). Characterization of spasticity in cerebral palsy: dependence of catch angle on velocity. Developmental Medicine and Child Neurology, 52, 563-569.

\section{TABLES}

Table 1 Psychometric properties (Mokkink et al., 2010) and their order of testing in the current study.

\begin{tabular}{|c|c|c|}
\hline Property & Definition & Analysis \\
\hline 1. Between-session reliability & $\begin{array}{l}\text { Establishes the capability of an } \\
\text { instrument to consistently } \\
\text { measure a variable between } \\
\text { sessions. }\end{array}$ & $\begin{array}{l}\text { Subjects were assessed } \\
\text { twice within four weeks. }\end{array}$ \\
\hline 2. Construct validity & $\begin{array}{l}\text { Establishes the ability of an } \\
\text { instrument to measure an } \\
\text { abstract construct and the } \\
\text { degree to which the instrument } \\
\text { reflects the theoretical } \\
\text { components of the construct. }\end{array}$ & $\begin{array}{l}\text { Inter-parameter correlations } \\
\text { were established between all } \\
\text { parameters. }\end{array}$ \\
\hline 3. Clinical validity & $\begin{array}{l}\text { Establishes if an instrument is } \\
\text { related to a commonly used } \\
\text { clinical method. }\end{array}$ & $\begin{array}{l}\text { Parameters of all signals } \\
\text { were compared to the } \\
\text { Modified Tardieu Scale. }\end{array}$ \\
\hline 4. Face validity & $\begin{array}{l}\text { The degree to which an } \\
\text { instrument indeed looks as an } \\
\text { adequate reflection of the } \\
\text { construct to be measured. }\end{array}$ & $\begin{array}{l}\text { Selected parameters were } \\
\text { used to visually categorize } \\
\text { the muscles by severity. }\end{array}$ \\
\hline
\end{tabular}

Table 2 Subjects' characteristics

\begin{tabular}{lll}
\hline & Subjects $(\mathbf{n}=46)$ & Subjects reliability study $(\mathbf{n}=12)$ \\
\hline Age (SD) & $9.07 \mathrm{yrs}(3.26 \mathrm{yrs})$ & $9.86 \mathrm{yrs}(3.1 \mathrm{yrs})$ \\
Mass (SD) & $29.10 \mathrm{~kg}(11.86 \mathrm{~kg})$ & $29.26 \mathrm{~kg}(10.27 \mathrm{~kg})$
\end{tabular}


Gender $($

MAS (range 0-3)

$\begin{array}{llll}\text { GAS }(\mathrm{n}) & \text { MEH }(\mathrm{n}) & \text { GAS }(\mathrm{n}) & \text { MEH (n) } \\ 1(2) & 0(3) & 1(2) & 1(4) \\ 1+(11) & 1(8) & 1+(3) & 1+(5) \\ 2(25) & 1+(11) & 2(5) & 2(2) \\ 3(8) & 2(22) & 3(2) & 3(1) \\ & 3(2) & & \end{array}$

$\begin{array}{lllll}\text { Mean MTS (SD) } & \text { GAS }(\mathrm{n}=44) & \text { MEH }(\mathrm{n}=35) & \text { GAS }(\mathrm{n}=10) & \text { MEH }(\mathrm{n}=8) \\ & -10.57^{\circ}\left(8.77^{\circ}\right) & -78.00^{\circ}\left(10.52^{\circ}\right) & -13.50^{\circ}\left(7.47^{\circ}\right) & -72.50^{\circ}\left(6.55^{\circ}\right) \\ \text { GMFCS-level } & \text { Range I }- \text { IV } & \text { Range I }- \text { III } & \\ & \text { I (17), II (17), III (10), IV (2) } & \text { I (5), II (6), III (1) } \\ \text { Diagnosis } & 19 \text { hemiplegia (7 left, 12 right) } & 8 \text { hemiplegia (3 left, } 5 \text { right }) \\ & 23 \text { diplegia } & 4 \text { diplegia } \\ & 3 \text { quadriplegia } & \end{array}$

MAS: Modified Ashworth Score; MTS: Modified Tardieu Score; GMFCS: Gross Motor Function Classification System (Palasino et al., 1997).

Table 3 Outcome parameters of GAS and MEH in both sessions (test, retest), with between session. Intraclass Correlation Coefficients (ICC), standard error of measurement (SEM), percentage of the SEM relative to the mean of the test and re-test values (\%SEM).

\begin{tabular}{lllllc}
\hline GAS & Test mean $($ SD) & Retest mean (SD) & ICC & SEM & \%SEM \\
\hline Max. dec. $\left({ }^{\circ} / \mathrm{sec}^{2}\right)$ & $1436.36(252.58)$ & $1407.14(268.13)$ & 0.42 & 228.45 & 16.02 \\
Max. dT/dt (Nm/s) & $133.66(54.63)$ & $119.33(40.66)$ & 0.88 & 22.20 & 17.55 \\
Work (J) & $3.80(1.30)$ & $4.19(1.26)$ & 0.68 & 0.92 & 23.08 \\
Min. Power (W) & $4.29(1.67)$ & $3.26(1.88)$ & 0.78 & 2.34 & 61.99 \\
RMS-EMG $(\%)$ & $5.66(4.65)$ & $8.19(8.01)$ & 0.55 & 5.09 & 73.52 \\
AOC1 $(\%)$ & $60.70(13.09)$ & $57.66(10.79)$ & 0.75 & 7.90 & 13.35 \\
\hline
\end{tabular}


COMPREHENSIVE QUANTIFICATION OF THE SPASTIC CATCH

\begin{tabular}{lllllc}
\hline AOC2 (\%) & $52.80(10.06)$ & $50.99(8.54)$ & 0.62 & 7.20 & 12.04 \\
AOC3 (\%) & $71.28(16.67)$ & $73.56(9.55)$ & 0.81 & 7.42 & 10.25 \\
MEH & & & & & \\
\hline Max. dec. $\left(\% / \mathrm{sec}^{2}\right)$ & $2237.76(549.09)$ & $1961.94(422.12)$ & 0.63 & 342.97 & 16.33 \\
Max. dT/dt (Nm/s) & $103.96(49.28)$ & $95.01(42.12)$ & 0.92 & 19.24 & 19.33 \\
Work (J) & $6.62(3.79)$ & $7.33(3.16)$ & 0.84 & 1.80 & 25.81 \\
Min. Power (W) & $7.29(5.05)$ & $4.84(2.61)$ & 0.54 & 4.56 & 75.19 \\
RMS-EMG (\%) & $17.90(32.64)$ & $18.44(33.68)$ & 0.99 & 2.93 & 16.13 \\
AOC1 (\%) & $81.45(10.40)$ & $77.90(9.53)$ & 0.71 & 6.76 & 8.48 \\
AOC2 (\%) & $45.67(28.00)$ & $47.13(25.00)$ & 0.72 & 15.77 & 33.99 \\
AOC3 (\%) & $88.59(11.24)$ & $87.64(10.27)$ & 0.86 & 5.40 & 6.13 \\
\hline
\end{tabular}

Max. dec.: maximum deceleration; Max. dT/dt: maximum change in torque; Min. Power: minimum power; RMS-EMG: root mean square envelope of surface electromyography; AOC: angle of catch.

Table 4 Spearman rank correlation coefficients between parameters for GAS and MEH.

\begin{tabular}{|c|c|c|c|c|c|c|c|c|c|}
\hline GAS & Max.dec & Max.dT/dt & Work & Min.Power & RMS-EMG & AOC1 & AOC2 & AOC3 & MTS \\
\hline Max.dec & 1 & 0.02 & -0.16 & 0.06 & 0.08 & -20.00 & $-0.48^{*}$ & -0.10 & -0.03 \\
\hline Max.dT/dt & & 1 & $0.59 *$ & -0.04 & $-0.37 *$ & -0.14 & 0.08 & -0.12 & 0.09 \\
\hline Work & & & 1 & 0.25 & $-0.30 *$ & $-0.60 *$ & $-0.33 *$ & $-0.54 *$ & -0.09 \\
\hline Min. Power & & & & 1 & -0.26 & $-0.29 *$ & -0.24 & 0.01 & 0.29 \\
\hline RMS-EMG & & & & & 1 & 0.09 & -0.07 & -0.01 & -0.10 \\
\hline AOC1 & & & & & & 1 & $0.74 *$ & $0.87 *$ & 0.20 \\
\hline
\end{tabular}




\begin{tabular}{|c|c|c|c|c|c|c|c|c|}
\hline$\overline{\mathrm{AOC} 2}$ & & & & & & 1 & $0.64 *$ & 0.12 \\
\hline AOC3 & & & & & & & 1 & $0.31 *$ \\
\hline MTS & & & & & & & & 1 \\
\hline МЕН & & & & & & & & \\
\hline Max. dec 1 & -0.13 & $-0.32 *$ & $-0.58 *$ & 0.032 & 0.34 & 0.07 & 0.04 & -0.08 \\
\hline Max. dT/dt & 1 & $0.76^{*}$ & -0.25 & 0.15 & $-0.42 *$ & -0.07 & $-0.44 *$ & $-0.49 *$ \\
\hline Work & & 1 & 0.11 & 0.25 & $-0.68 *$ & -0.26 & $-0.57 *$ & $-0.36^{*}$ \\
\hline Min. Power & & & 1 & 0.22 & $-0.35^{*}$ & -0.25 & -0.12 & -0.08 \\
\hline RMS-EMG & & & & 1 & $-0.60 *$ & -0.25 & $-0.65 *$ & $-0.38 *$ \\
\hline $\mathrm{AOC} 1$ & & & & & 1 & $0.40 *$ & $0.88 *$ & 0.30 \\
\hline $\mathrm{AOC} 2$ & & & & & & 1 & $0.30^{*}$ & 0.13 \\
\hline AOC3 & & & & & & & 1 & $0.34 *$ \\
\hline MTS & & & & & & & & 1 \\
\hline
\end{tabular}

GAS: gastrocnemius; MEH: medial hamstrings; Max. dec: maximum deceleration; Max. dT/dt: maximum change in torque; Min. Power: minimum power; RMS-EMG: root mean square of surface electromyography; AOC: angle of catch; MTS: modified Tardieu Scale. 


\section{FIGURES}
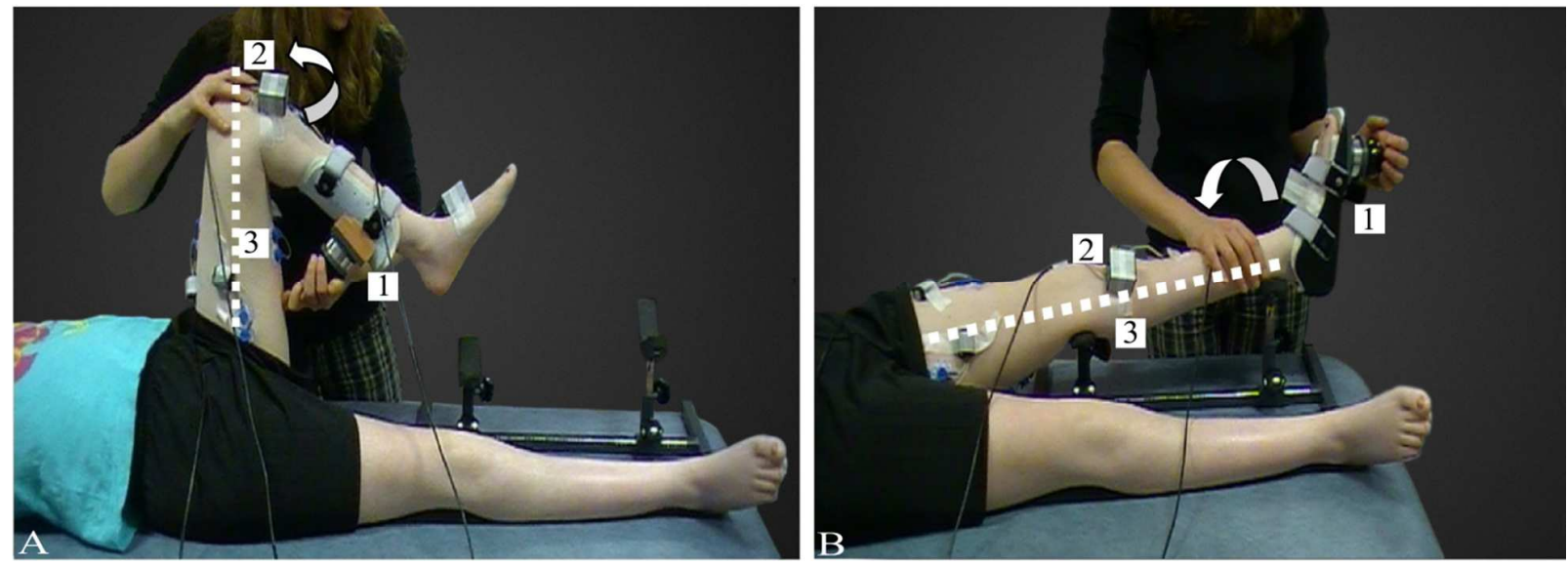

Figure 1 Test starting positions, direction of stretch (white arrows), and instrumentation for the objective spasticity assessments in A) the medial hamstrings (MEH) and B) the gastrocnemius (GAS). Overview of the test instrumentation: (1) a six degrees of freedom force-sensor attached to either a shank orthosis on the posterior aspect of the lower leg (MEH) or a foot orthosis (GAS), used to measure reactive torque; (2) two inertial measurements units measured joint angle characteristics; and (3) surface electromyography (sEMG) measured muscle activity of the agonistic and antagonistic muscle groups. 
COMPREHENSIVE QUANTIFICATION OF THE SPASTIC CATCH

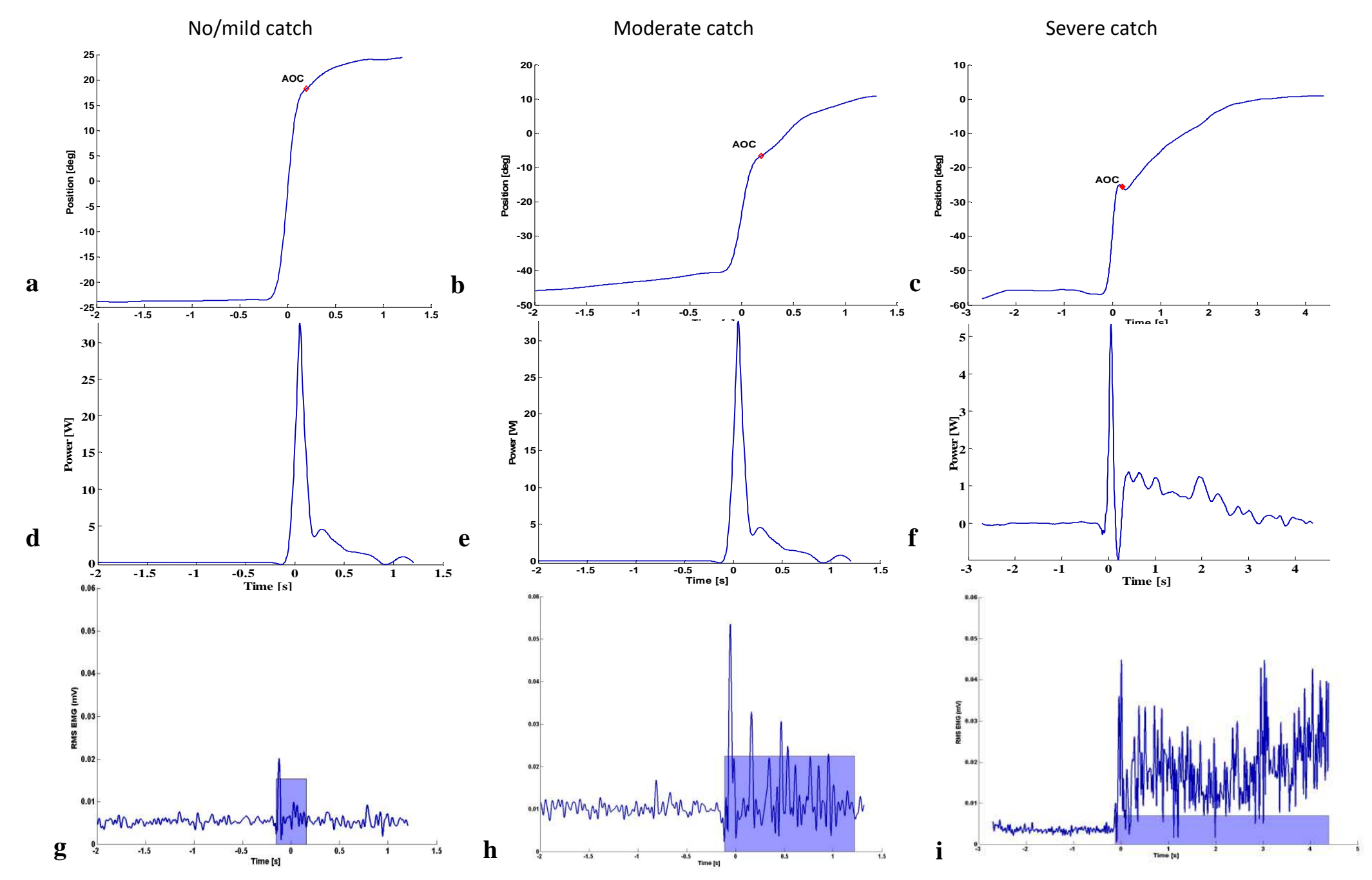


Figure 2 Example of position- (a-c), power- (d-f), and RMS-EMG- (g-i) time graphs depicting the integrated clinical interpretation of a mild (left), moderate (middle) and severe (right) catch during high velocity stretches to the gastrocnemius. Zero seconds indicates times of maximal velocity. The rectangle in the RMS-EMG-time graph indicates the duration of EMG onset. AOC: angle of catch; RMS-EMG: root mean square envelope of surface electromyography. 

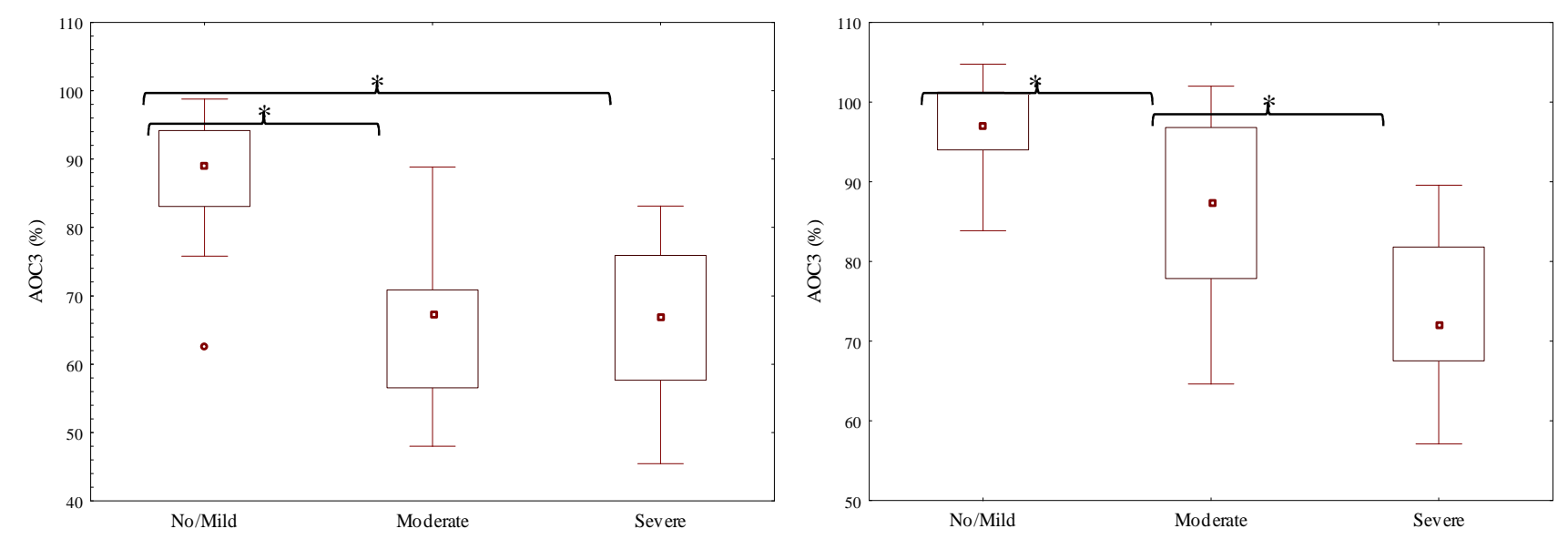

a

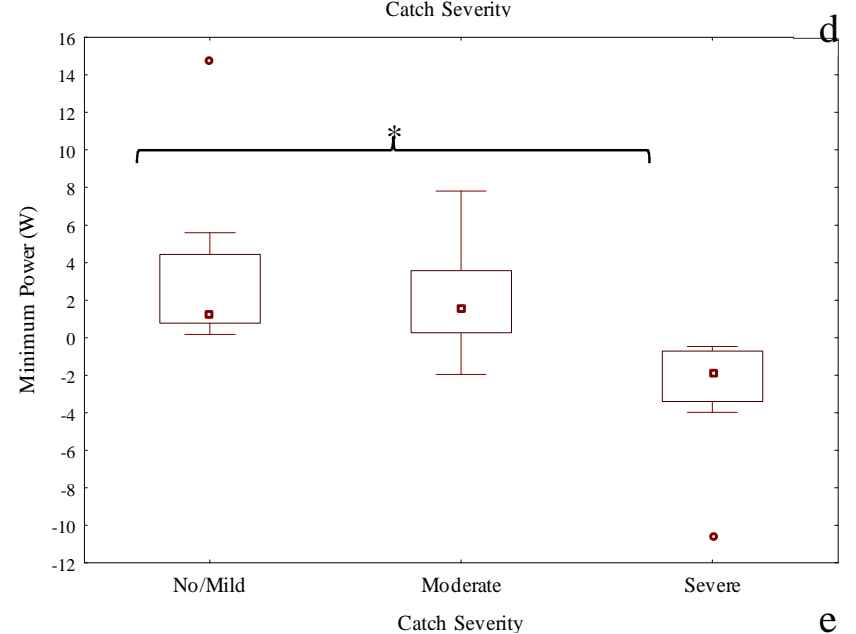

d

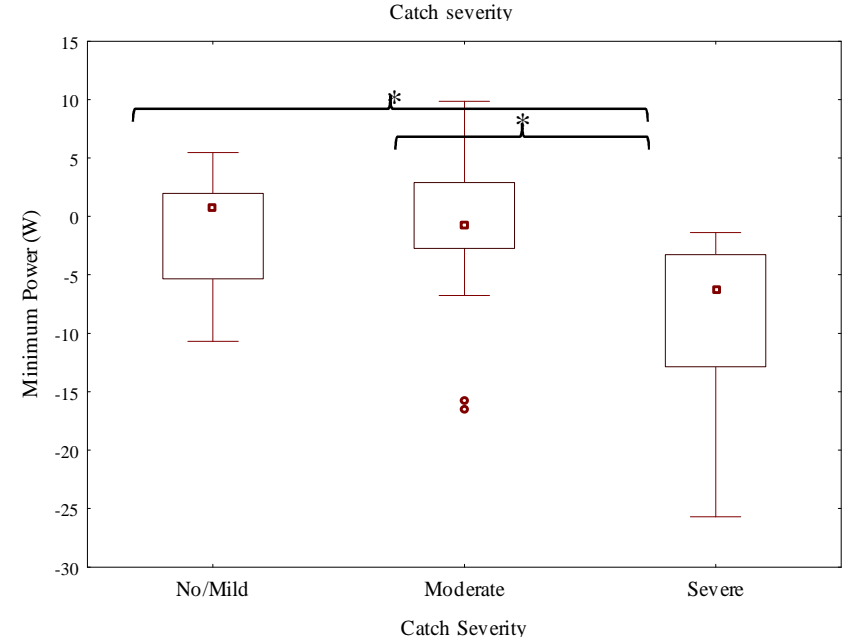

$\mathrm{f}$

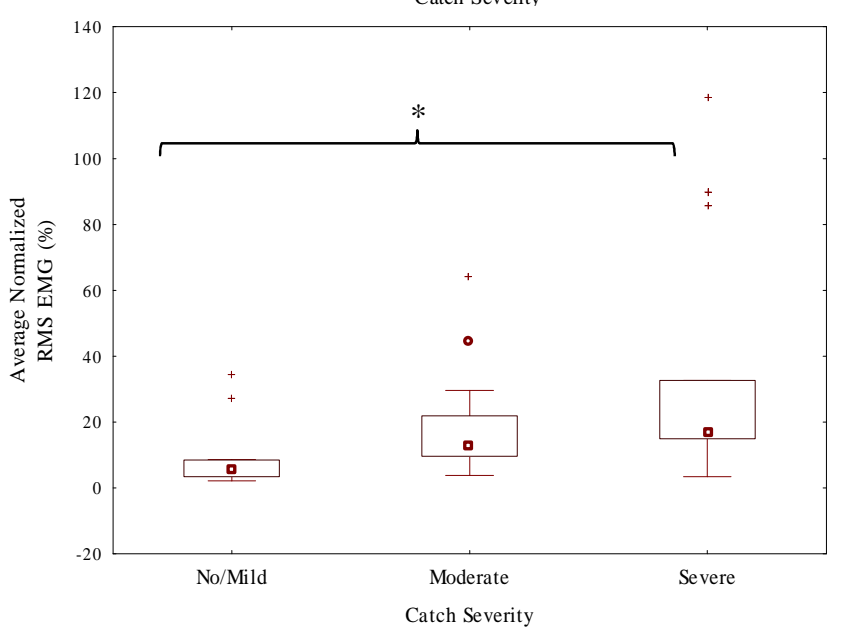

Median $\square$

$25 \%-75 \% \square$

Non-Outlier Range 工

Outliers o

Extrees + 
Figure 3. Box plots of selected severity-related parameters used for visual categorization of the (a-c) gastrocnemius and the (d-f) medial hamstrings. AOC: angle of catch; RMS-EMG: root mean square envelope of the surface electromyography. ${ }^{*} \mathrm{p}<0.05$, Man Whitney $U$ tests.

\section{APPENDIX A}
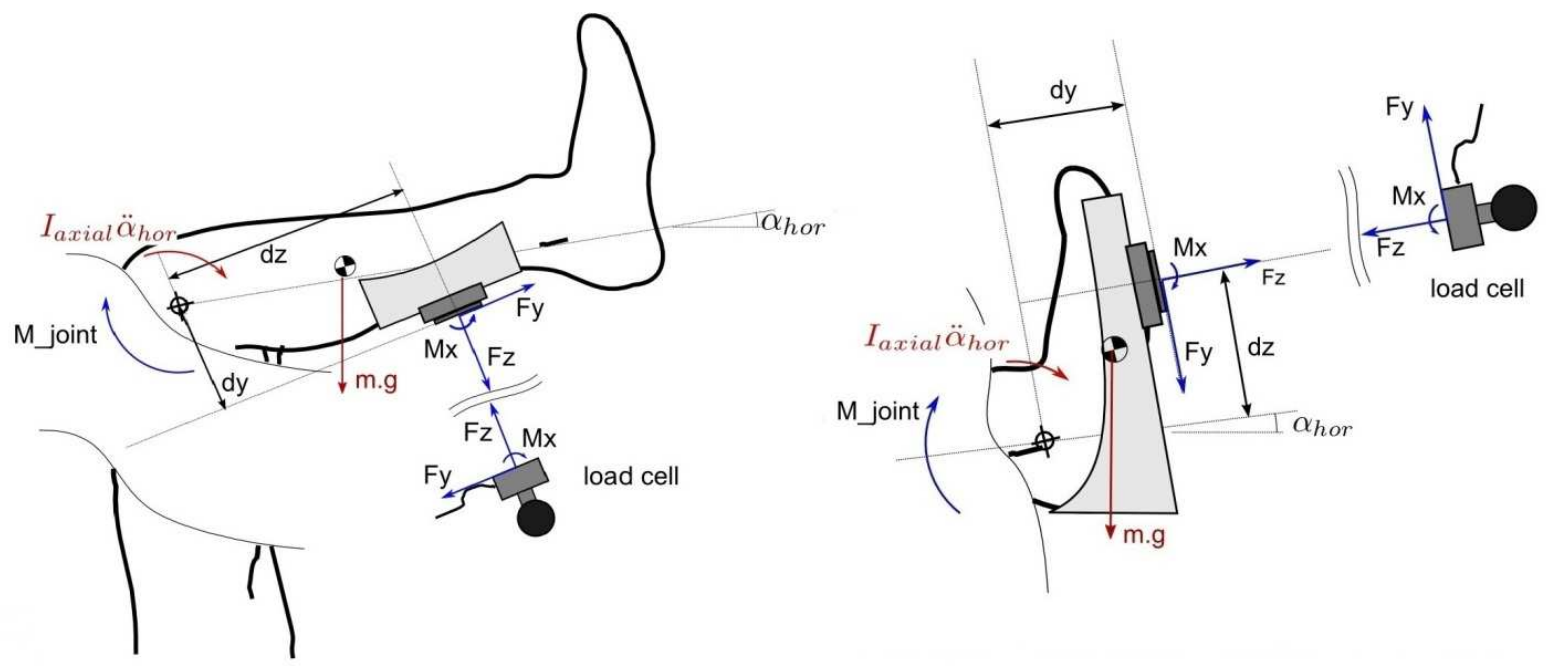

Figure 1 Free body diagram of medial hamstrings and gastrocnemius tests. The masses of the orthoses are considered negligible. $d_{y}$ and $d_{z}$ correspond to the distances measured by the therapist. The mass parameters of the shank and foot are determined by an anthropometric model (Jensen, 1986). The body segment is moved with an angular acceleration of $\alpha_{\text {hor }}$. The moments for the joints are given by:

$$
\begin{gathered}
\text { Eq (A.1) } \quad M_{\text {knee }}=-F_{z}+F_{y} D_{y}+M_{x}-m g \cos \left(\alpha_{\text {hor }}\right) r_{\text {prox }}-I_{\text {axial }} \ddot{\alpha}_{\text {hor }} \\
\text { Eq (A.2) } \quad M_{\text {ankle }}=-F_{z} d_{z}-F_{y} d_{y}-M_{x} \\
\left(\text { with } I_{\text {axial }}=m k_{\text {prox }}^{2}\right)
\end{gathered}
$$

Where $M_{-}$joint is the net internal moment acting at the joint and is composed of different components: the perpendicular force $\left(\mathrm{F}_{\mathrm{z}}\right)$, the non-perpendicular force components $\left(\mathrm{F}_{\mathrm{y}}\right)$, the 
COMPREHENSIVE QUANTIFICATION OF THE SPASTIC CATCH

torque exerted on the handle $\left(\mathrm{M}_{\mathrm{x}}\right)$, gravity (m.g) and inertia ( $\left.\mathrm{I}_{\text {axial }}\right)$. In Eq. A.2, the weight of the foot is considered negligible. 